\section{Percepción de académicos de carreras de la salud de Chile sobre el perfeccionamiento docente}

\author{
CRISTHIAN PÉREZ V. ${ }^{1, \mathrm{a}}$, EDUARDO FASCE H. ${ }^{1}$, KATHERINE COLOMA N. ${ }^{2, \mathrm{~b}}$, \\ GIULIETTA VACCAREZZA G. ${ }^{2, \mathrm{c}}$, JAVIERA ORTEGA B. ${ }^{1, \mathrm{~d}}$
}

\section{Perceptions about continuous training of Chilean health care teachers}

Background: Continuous training of teachers, in discipline and pedagogical topics, is a key step to improve the quality of educational processes. Aim: To report the perception of Chilean teachers of undergraduate health care programs, about continuous training activities. Material and Methods: Twenty teachers working at different undergraduate health care programs in Chile were interviewed. Maximum variation and theoretical sampling methods were used to select the sample. Data was analyzed by open coding, according to the Grounded Theory guidelines. Results: Nine categories emerged from data analysis: Access to continuous training, meaning of training in discipline, activities of continuous training in discipline, meaning of continuous training in pedagogy, kinds of continuous training in pedagogy, quality of continuous training in pedagogy, ideal of continuous training in pedagogy, outcomes of continuous training in pedagogy and needs for continuous training in pedagogy. Conclusions: Teachers of health care programs prefer to participate in contextualized training activities. Also, they emphasize their need of training in evaluation and teaching strategies.

(Rev Med Chile 2013; 141: 787-792). topic.

Key words: Education, medical; Faculty, medical; Programmed instruction as

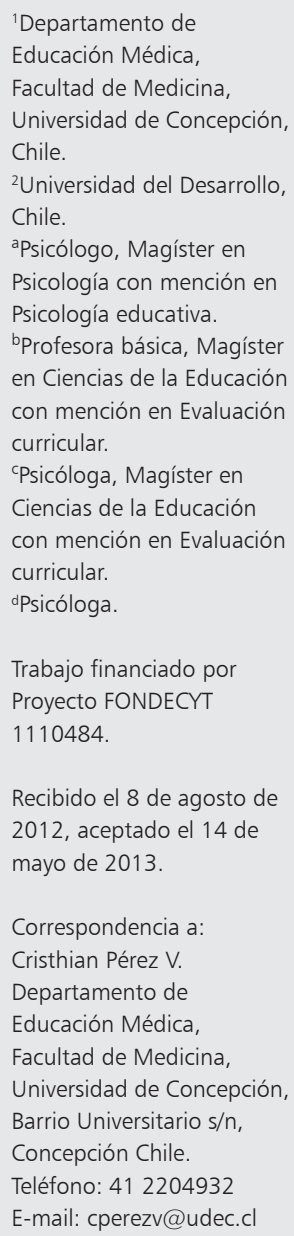

L a educación superior, clave para el desarrollo individual y nacional ${ }^{1,2}$, enfrenta múltiples desafíos; globalización, expansión y excesiva disponibilidad de información ${ }^{1,3-5}$, sumándose la demanda social por una formación más integral y pertinente ${ }^{1,6}$. Esto se acompaña de nuevos modelos curriculares en educación ${ }^{3,6,7}$ que están forzando al cambio de los procesos formativos en distintas áreas, incluyendo las carreras de la salud ${ }^{5}$.

Estos cambios apuntan directamente a la práctica docente. Ya en 1993 Lowry sentenciaba que aunque tradicionalmente se asumía que todo profesional podía enseñar, este nuevo contexto dificultaba que profesionales sin conocimientos en educación hicieran docencia ${ }^{8}$. Además, ser académico universitario implica asumir múltiples roles: investigador, coordinador, docente, etc. ${ }^{6} \mathrm{Y}$ de éstos, la docencia por sí sola es compleja, pues involucra planificar, proveer información, facilitar aprendizajes, evaluarlos, etc. ${ }^{9,10}$.

Asumir tan exigente función demanda, primero, dominio disciplinar en lo que se enseña ${ }^{11,12}$, y una forma de obtenerlo es desde la experiencia profesional, particularmente deseable en docentes del área de la salud ${ }^{5}$. Pero, igualmente, requiere capacitación formal continua, sobre todo cuando el mantenimiento de las competencias y el desarrollo profesional no son asegurados por el pregrado o las especialidades ${ }^{12,13}$.

Sin embargo, como apuntaba Lowry, la docencia requiere más que saber qué enseñar (dominio disciplinar): demanda saber cómo (dominio pedagógico). Adquirir competencias técnicas para enseñar es la única forma de asumir las numerosas 
tareas de la docencia en un escenario donde ya no es útil replicar viejos modelos $^{14}$, ni es plausible esperar volverse competente de forma autodidac$\mathrm{ta}^{15}$ : Se requieren procesos de formación inicial y continua en educación para entrenar a quienes practican la docencia universitaria ${ }^{15,16}$. Esto es más apremiante para aquellos académicos sin formación en educación durante su pregrado -la mayoría-, pues enfrentan las dificultades de la docencia sin herramientas básicas para enseñar ${ }^{17}$.

Lamentablemente, aunque el perfeccionamiento pedagógico es una necesidad reconocida en ciencias de la salud, tanto como la experiencia profesional y el dominio disciplinar ${ }^{5,14}$, los incentivos por capacitarse en el tema son limitados.

Aunque la docencia es el eje del proceso formativo, históricamente ha sido devaluada frente a otros roles como el investigativo, que recibe mayores recompensas y financiamientos ${ }^{15,18}$.

Por ello, universidades como Harvard han tomado medidas para incentivar la buena docen$\mathrm{cia}^{18}$. Pero, en Latinoamérica la falta de estímulos merma el aporte de la educación médica: Por ejemplo, muchas de las estrategias pedagógicas que se promueven para carreras de la salud, como el Aprendizaje Basado en Problemas (ABP) y la Evaluación Clínica Objetiva Estructurada $(\mathrm{ECOE})^{19}$, mantienen una implementación limitada ${ }^{5}$.

En Chile, el compromiso por el desarrollo docente se ha asumido en diversas carreras del área de la salud, surgiendo programas de formación pedagógica, inclusive en postgrado ${ }^{14}$. Pero otro de los riesgos que afectan al perfeccionamiento pedagógico es la calidad de la oferta. Usualmente este tipo de especialización suele producirse fuera de los campos disciplinares de los docentes, reforzando la imagen de que sus contenidos les son ajenos. En consecuencia, considerar el campo disciplinar específico de cada docente, y el contexto donde se enseña,es vital para comprender y mejorar sus prácticas ${ }^{17}$, sobre todo cuando compartir experiencias y problemas comunes es uno de los motivadores para realizar perfeccionamientos pedagógicos ${ }^{20}$.

Otra amenaza corresponde a las estrategias de capacitación, que desconocen que los destinatarios son adultos con altos niveles instruccionales y usualmente recurren a modelos más pedagógicos (centrados en transmitir contenidos a alumnos dependientes) que andragógicos (orientados a facilitar la construcción autónoma de aprendi- zajes $)^{7,21}$. Esto decepciona a los asistentes, y los hace evaluar los contenidos como irrelevantes y a estas instancias como incompatibles con el propio estilo docente, generando una mayor resistencia a participar $^{22}$.

En contraposición, estudios realizados sobre programas de perfeccionamiento específicos para académicos de la salud aplicando métodos andragógicos, han mostrado generar alta satisfacción con el programa y sus profesores, cambios favorables en las actitudes docentes, percepción de haber aprendido y un positivo impacto atribuido en el desempeño docente ${ }^{23}$.

Ante esto, el presente estudio busca abordar el tema del perfeccionamiento de los docentes del área de la salud, describiendo las experiencias de perfeccionamiento de éstos.

Lo anterior, como una forma de disminuir una deuda de la educación médica, que pese a su relativa madurez como disciplina ${ }^{24}$, ha centrando sus esfuerzos investigativos en estudiantes e innovaciones pedagógicas, con escasa atención alas variables del docente, el otro actor clave del proceso formativo ${ }^{10}$. Esto, pese a distintas organizaciones latinoamericanas que establecen que la evaluación de las capacidades docentes es una línea de investigación necesaria de desarrollar ${ }^{5}$.

\section{Metodología}

Este estudio cualitativo aplicó la Grounded Theory ${ }^{25}$ según los lineamientos de Strauss y Corbin $^{26}$.

Veinte docentes de carreras de la salud,de las regiones del Maule, Bío-Bío y Araucanía, fueron seleccionados mediante muestreo de máxima variación como arranque muestra ${ }^{27}$ y muestreo teórico para el cierre muestral, hasta alcanzar la saturación ${ }^{26}$. Tenían entre 5 y 40 años de ejercicio docente en programas de Medicina, Enfermería, Fonoaudiología, Nutrición y Dietética, Odontología, Kinesiología y Tecnología Médica. Catorce eran mujeres. Doce trabajaban en universidades privadas.

Los participantes respondieron entrevistas temáticas ${ }^{28}$, semiestructuradas, que facilitan la comparación y profundización de información producida $^{29}$, previo consentimiento informado. Los investigadores y ayudantes capacitados realizaron las entrevistas como un diálogo flexible, aunque ceñido a un guión temático que abordaba: 
tipos de perfeccionamiento, temas abordados, participación en éstos, evaluación de la instancia y resultados obtenidos.

El estudio fue aprobado por los Comités de Ética de las Universidades ejecutoras, y visado por el Comité Asesor de Bioética de CONICYT.

El análisis empleó codificación abierta, según la Grounded Theor ${ }^{26}$, identificando y describiendo inductivamente categorías de análisis, esto es núcleos conceptuales que emergen del discurso. Se aplicó triangulación entre analistas.

\section{Resultados}

Desde la codificación abierta emergieron nueve categorías, descritas a continuación. Éstas se ilustran con viñetas textuales, identificando al informante (número romano) y al párrafo de la cita (número arábigo).

\section{Acceso al perfeccionamiento}

Los docentes coinciden en que las universidades se interesan y promueven el perfeccionamiento académico tanto disciplinar como pedagógico, facilitando el acceso a éste, apoyando financieramente o generando iniciativas de capacitación propias. Estas últimas, principalmente en temáticas educativas, serían más evidentes en universidades privadas.

"nunca tuve ningún problema en relación a quién se iba a hacer cargo, quién iba a cumplir mis horas [...], dan todas esas facilidades. Incluso dan un apoyo económico importante en relación al logro de esta especialización" (XIV 305).

No obstante, estas oportunidades se distribuyen desigualmente, pues estarían disponible preferente-y en algunos casos, exclusivamente- para profesores con contrato estable.

\section{Significado del perfeccionamiento disciplinar}

La especialización disciplinar constante se entiende como una necesidad de desarrollo personal, pero principalmente como una obligación del rol docente, fundamentalmente en los tópicos en que hace docencia. Un dominio más profundo de los contenidos es reconocido como requisito básico para enseñar y facilitaría la transmisión de conocimientos.

El dominio disciplinar mínimo para hacer docencia sería más difícil de alcanzar en la actualidad, pues reconocen que el conocimiento científico avanza aceleradamente, los alumnos acceden rápidamente a la información y el pregrado es insuficiente para alcanzar la experticia que requieren los académicos para ejercer su rol.

Pero, la importancia atribuida al perfeccionamiento disciplinar va disminuyendo en la medida en que el docente se forma en educación, pues va asumiendo que el contenido no es todo.

“... mi principal inquietud era [al inicio] eso: llegar al punto de dominar la materia lo suficientemente bien para sentirme cómoda con cualquier método de enseñanza. Pero no me cuestionaba el método, me cuestionaba el contenido" (III 34).

\section{Actividades de perfeccionamiento disciplinar}

Los docentes refieren haber participado en diversas actividades de especialización en salud, abordando tanto temáticas de ciencias básicas como aplicadas. Pocos reportan especializaciones médicas, postgrados y pasantías en el extranjero, pero la capacitación continua en cursos, congresos y actividades acotadas es común a los informantes.

La evaluación de las instancias es diversa. Algunos docentes, más que los aprendizajes logrados, destacan la oportunidad de conocer otros profesionales y experimentar los programas como alumnos, lo que hace cuestionar la formación en salud.

Además de las instancias formales, reconocen a la experiencia profesional y la misma autoformación como instancias válidas de perfeccionamiento disciplinar.

"aplico mucho lo que fue mi experiencia profesional, previo a dedicarme a la labor docente. No soy de los profesionales que inmediatamente salió y entró a hacer docencia" (VII 162)

4. Significado del perfeccionamiento pedagógico: La visión del perfeccionamiento en ésta área varía entre quienes lo consideran esencial para ejercer la docencia y quienes sólo lo entienden como un complemento positivo.

"Yo creo que siempre es bueno tener formación pedagógica, [pero] no creo que todos los profesores universitarios tengan que tener un magister en pedagogía” (XIV 305).

No obstante, quienes concuerdan con esta última visión reportan experiencias previas negativas en perfeccionamientos educativos, percibiéndolos como excesivamente teóricos y descontextualizados. 


\section{Formas de perfeccionamiento pedagógico}

Los informantes han participado en alternativas formales e informales. Entre las primeras cuentan cursos, diplomados y postgrados disponibles, destacando aquellos dirigidos específicamente a académicos del área. Entre las segundas se percibe la propia experiencia como vía de especialización, los modelos (y anti-modelos) docentes que incluyen a los pares o los profesores que se tuvo en pregrado o en enseñanza media, el apoyo de los pares y la autoformación.

"... soy autoformado en todo, en pedagogía igual, lo que tiene que hacer uno es mantener el interés en algo, buscar lo que hay sobre ese algo y buscar lo relevante" (X 98).

Las temáticas abordadas en estas instancias son variadas, incluyendo teorías, planificación, didáctica, evaluación, curriculum, entre otras. No obstante, independiente de la temática siempre se considera que el tiempo destinado es insuficiente para dominarla.

\section{Calidad del perfeccionamiento pedagógico}

Las estrategias de capacitación experimentadas por los docentes también son variadas, desde clases expositivas hasta talleres prácticos y asesorías personalizadas. Entre éstas, son especialmente críticos con las actividades teóricas y aquellas que reúnen a docentes de distintas áreas del conocimiento: Las describen como descontextualizadas y de utilidad incierta, pues el dominio de los contenidos y su aplicabilidad a la propia docencia no serían adecuadamente dirigido ni supervisado. Además, no reconocerían el perfil del docente que asiste a las capacitaciones: heterogéneo en sus disciplinas de origen, sus estilos de aprendizaje y su dominio de la materia.

En contraposición, valoran las actividades prácticas, que implican trabajo guiado y que llevan a reflexionar e innovar en lo que ya se está haciendo en docencia.

"es fácil cuando tú vas por ejemplo con una rúbrica: échele una miradita a mi rúbrica, ¿estará buena?, ¿estará mala?...y ahí he aprendido harto..." (XXI 170).

\section{Ideal de capacitación pedagógica}

Asociado a lo anterior, el ideal de capacitación en temáticas educativas es aquella que recurre a diversas herramientas didácticas, incluyendo, aunque no restringiéndose, a clases expositivas.
Asimismo se valora la oportunidad de poder intercambiar experiencias con profesionales de otras carreras sanitarias, ampliando la concepción de la docencia. Sin embargo, la clave para lograr aprendizajes sería realizar trabajos contextualizados en la docencia que efectivamente se está realizando, supervisados estrechamente por especialistas y que deriven en productos tangibles.

"Se mezcla lo que es una explicación, con trabajo, con discusión, con análisis eh... [...] y después a la salida con algún producto" (II 300).

Asimismo, se enfatiza la necesidad de seguimiento posterior a la capacitación, porque la lejanía que los docentes tienen con los contenidos educacionaleslos hace sentir inseguros de poder implementar los nuevos aprendizajes sin guía y corrección constante.

\section{Resultados del perfeccionamiento pedagógico}

Dentro de los principales logros asociados se destaca que los buenos perfeccionamientos motivan a seguir especializándose en el área y amplían la visión de la propia labor, al aumentar la comprensión del proceso enseñanza-aprendizaje y de las variables influyentes. Esto les ayudaría a flexibilizarse, buscando estrategias para adaptarse a la diversidad del estudiantado.

También, las capacitaciones permiten acceder a experiencias de otros docentes, retroalimentando la práctica personal.

En términos técnicos, algunos informantes sostienen que el perfeccionamiento permite organizar y fundamentar las prácticas que intuitivamente realizan. Igualmente, se destacan aprendizajes logrados en planificación, estrategias de enseñanza, manejo de aula y evaluación, entre los cuales ésta última sobresale como la más compleja, porque implica cambios en la forma de pensarla y en los criterios utilizados para construir los instrumentos con que se aplica.

Los cambios derivados de estos aprendizajes, cuando son profundos, involucrarían una transformación en la forma de hacer docencia, siendo visible para el mismo docente, sus alumnos y los demás académicos.

"[Mi curso] yo lo diseñé totalmente diferente a partir de lo que aprendi en el magister. Por ejemplo, antes sólo hacía clases así como teóricas, típicas, magistrales entre comillas, y ahora cambié totalmente $y$, por ejemplo, antes de partir el año planifico todas las clases" (VIII 18). 


\section{Necesidades de perfeccionamiento pedagógico}

En general, los docentes quisieran asistir a todo tipo de capacitación en educación, pero las mayores necesidades se dan en el área de las estrategias de enseñanza y la evaluación. La primera, aunque no se percibe como la más débil sería potenciada y sistematizada por la formación pedagógica. Los docentes se perciben como creativos para idear estrategias de enseñanza, pero creen que la capacitación les sumaría opciones.

Sería la evaluación la principal debilidad docente, requiriéndose capacitación para diseñar procesos de evaluación coherentes con los objetivos y las estrategias de enseñanza, y para construir instrumentos evaluativos. A esta área, asimismo, se le atribuye una alta complejidad técnica.

"o sea yo creo que la gente evalúa porque.... porque tiene que poner nota, pero en el fondo no tiene idea lo que esta evaluando" (IX 63).

Secundariamente, también emerge en aquellos docentes que han cursado postgrados en educación la necesidad de capacitarse en investigación educativa para indagar en la docencia universitaria. En esta área, en general se atribuyen falta de conocimiento y experiencia básica.

\section{Discusión}

El estudio describe una clara preocupación por recurrir a instancias formales de capacitación pedagógica, donde las herramientas evaluativas son la principal demanda, pues se le atribuye una alta complejidad técnica.

Lo anterior contrasta con una menor preocupación por la enseñanza, alertando de una eventual sobrevaloración de las propias competencias didácticas, porque estarían vinculando la capacidad de enseñar más al dominio disciplinar que al manejo de estrategias pedagógicas. Y esto es preocupante, pues son competencias diferentes y el escenario actual exige un dominio pedagógico importante en docentes de todas las áreas, incluidas las ciencias de la salud ${ }^{8,14,15}$.

Conjuntamente, aparece un cuestionamiento a la calidad de la oferta en perfeccionamiento, criticándose a aquellas instancias excesivamente teóricas, no focalizadas en la disciplina y cuya aplicación práctica queda reservada a las habilidades de los asistentes.

Esto se contrapone con aquellas dimensiones propias de la andragogía $a^{7,20}$, principalmente con el aprendizaje experiencial y la aplicabilidad de lo aprendido; que apuntan a la resolución de problemáticas propias de la cotidianeidad del adulto, explicando que los docentes busquen herramientas aplicables inmediatamente y orientadas a la solución de problemas, más que a la construcción de modelos teóricos o hipotéticos.

Lo anterior, congruente con la positiva evaluación de experiencias andragógicas en Chile ${ }^{23}$, enfatiza la importancia de una buena elección de estrategias en estas capacitaciones, pues influyen en los aprendizajes logrados y su transferencia al aula, pero además en el interés y motivación por perfeccionarse de quienes hacen docencia.

\section{Referencias}

1. Hernández AC. Los desafíos de la docencia universitaria. Revista Educación de la Universidad de Costa Rica 2002; 26 (2): 117-24.

2. Ruiz L, Pachano L. La docencia universitaria y las prácticas evaluativas. La revista venezolana de educación 2005; 9 (31): 531-40.

3. Fasce E. Los nuevos roles del docente de Medicina. Rev Educ Cienc Salud 2004; 1: 7-13.

4. García Y. Nuevos desafíos bioéticos en la formación de los profesionales de la salud: reflexiones a partir de la experiencia en el Espacio Europeo de Educación Superior (EEES). Rev Educ Cienc Salud 2011; 8:83-88.

5. González-Martínez JF, García-García JA, MendozaGuerrero JA, Uriega-González S. La importancia de la investigación en educación médica en México. Rev Med Hosp Gen Mex 2010; 73 (1): 48-56.

6. Álvarez-Rojo V, Asensio-Muñoz I, Clares J, Del-Frago R, García-Lupión B, García-Nieto N, et al. Perfiles docentes para el espacio europeo de educación superior (EEES) en el ámbito universitario español. Relieve 2009;15 (1): 1-18.

7. Fasce E. Andragogía. Rev Educ Cienc Salud 2006; 3(2):69.

8. Lowry S. Teaching the teachers. BMJ 1993; 306: 127-30.

9. Harden RM, Crosby J. AMEE Guide No 20: The good teacher is more than a lecturer - the twelve roles of the teacher. MedTeach2000; 4: 334-47.

10. Martínez-González A, López-Bárcena J, Herrera P, Ocampo-Martínez J, Petra I, Uribe-Martínez G, et al. Modelo de competencias del profesor de medicina. Educmed 2008; 11 (3): 157-67.

11. Cabalín D, Navarro N. Conceptualización de los Estudiantes sobre el Buen Profesor Universitario en las 
Carreras de la Salud de la Universidad de La FronteraChile. Int J Morphol 2008; 26 (4): 887-92.

12. Kunakov N, Romero L. Las características esperadas del docente en una innovación curricular orientada a competencias. Revista de docencia universitaria 2012; 10 (especial): 257-68.

13. Larre U, Petruccelli D, Niski R, Fosman E, Amoza B, Margolis A, et al. El desarrollo profesional continuo en el Uruguay de cara al siglo XXI. Rev Panam Salud Pública 2003; 13 (6): 410-8.

14. Fasce E. ¿Son suficientes las competencias profesionales en la enseñanza de las ciencias de la salud?. Rev Educ Cienc Salud 2008; 5(2):81.

15. Montero L, Triviño X, Sirhan M, Moore P, Leiva L. Barreras para la formación en docencia de los profesores de medicina: una aproximación cualitativa. Rev Med Chile 2012, 140: 695-702.

16. Imbernón F. Responsabilidad social, profesionalidad y formación inicial en la docencia universitaria. Revista interuniversitaria de formación del profesorado 1999; 34: 123-32.

17. Escudero T. La formación pedagógica del profesorado universitario vista desde la enseñanza disciplinar. Revista de Educación 2003; 331: 101-21.

18. Sirhan M, Triviño X. Evaluación de una experiencia de capacitación en planificación educacional para directores de programas de las especialidades médicas. Rev Med
Chile 2012; 140: 530-7.

19. Villa A. La excelencia docente. Revista de Educación 2008; número extraordinario: 177- 212.

20. Martínez JM. Los métodos de evaluación de la competencia profesional: la evaluación clínica objetivo estructurada (ECOE).Educmed 2005; 8 (2): 18-22.

21. Knowles M, Holton F, Swanson R. Andragogía: el aprendizaje de los adultos. Mexicana. México 2001.

22. Beavers A. Teachers as learners: Implications of adult education for professional development. Journal of college teaching and learning 2009; 6 (7): 25-30.

23. Triviño X, Sirhan M, Moore P, Montero L. Impacto de un programa de formación en docencia en una escuela de medicina. Rev Med Chile 2011; 139: 1508-15.

24. Gual A. La educación médica en Latinoamérica. Educmed 2009; 12 (1): 1-2.

25. Glaser B, Strauss A. The discovery of Grounded Theory. Aldine Transaction. New Jersey 1967.

26. Strauss A, Corbin J. Bases de la investigación cualitativa. Universidad de Antioquía. Medellín 2002.

27. Rodríguez G, Gil J, García E. Metodología de la Investigación Cualitativa. Aljibe. Málaga 1999.

28. Flick U. Introducción a la Investigación Cualitativa. Morata. Madrid 2004.

29. Vieytes R. Metodología de la investigación en organizaciones, mercado y sociedad. Editorial de las Ciencias. Buenos Aires 2005. 